\title{
Effects of habitat composition and landscape structure on worker foraging distances of five bumble bee species
}

\author{
John W. Redhead, ${ }^{1,5}$ Stephanie Dreier,,${ }^{2,3}$ Andrew F. G. Bourke, ${ }^{4}$ Matthew S. Heard, ${ }^{1}$ William C. Jordan, ${ }^{2}$ \\ Seirian Sumner, ${ }^{2,3}$ Jinliang Wang, $^{2}$ and Claire Carvell ${ }^{1}$ \\ ${ }^{1}$ NERC Centre for Ecology and Hydrology, Benson Lane, Crowmarsh Gifford, Wallingford, OX108BB UK \\ ${ }^{2}$ Institute of Zoology, Zoological Society of London, Regent's Park, London, NW14RY UK \\ ${ }^{3}$ School of Biological Sciences, Bristol Life Sciences Building, University of Bristol, 24 Tyndall Avenue, Bristol BS81TQ UK \\ ${ }^{4}$ School of Biological Sciences, University of East Anglia, Norwich Research Park, Norwich, NR47TJ UK
}

\begin{abstract}
Bumble bees (Bombus spp.) are important pollinators of both crops and wildflowers. Their contribution to this essential ecosystem service has been threatened over recent decades by changes in land use, which have led to declines in their populations. In order to design effective conservation measures, it is important to understand the effects of variation in landscape composition and structure on the foraging activities of worker bumble bees. This is because the viability of individual colonies is likely to be affected by the trade-off between the energetic costs of foraging over greater distances and the potential gains from access to additional resources. We used field surveys, molecular genetics, and fine resolution remote sensing to estimate the locations of wild bumble bee nests and to infer foraging distances across a $20-\mathrm{km}^{2}$ agricultural landscape in southern England, UK. We investigated five species, including the rare B. ruderatus and ecologically similar but widespread $B$. hortorum. We compared worker foraging distances between species and examined how variation in landscape composition and structure affected foraging distances at the colony level. Mean worker foraging distances differed significantly between species. Bombus terrestris, B. lapidarius, and B. ruderatus exhibited significantly greater mean foraging distances $(551,536$, and $501 \mathrm{~m}$, respectively) than B. hortorum and B. pascuorum (336 and $272 \mathrm{~m}$, respectively). There was wide variation in worker foraging distances between colonies of the same species, which was in turn strongly influenced by the amount and spatial configuration of available foraging habitats. Shorter foraging distances were found for colonies where the local landscape had high coverage and low fragmentation of seminatural vegetation, including managed agri-environmental field margins. The strength of relationships between different landscape variables and foraging distance varied between species, for example the strongest relationship for B. ruderatus being with floral cover of preferred forage plants. Our findings suggest that management of landscape composition and configuration has the potential to reduce foraging distances across a range of bumble bee species. There is thus potential for improvements in the design and implementation of landscape management options, such as agri-environment schemes, aimed at providing foraging habitat for bumble bees and enhancing crop pollination services.
\end{abstract}

Key words: agri-environment; Bombus; foraging range; landscape scale; pollination; spatial ecology; wild colonies.

\section{INTRODUCTION}

Bumble bees are important pollinators of wild plants (Holzschuh et al. 2011, Kovács-Hostyánszki et al. 2013) and a range of crops (Garratt et al. 2014). Along with other wild and managed pollinators, they provide an essential ecosystem service, affecting the stability of natural ecosystems as well as agricultural productivity. Many bumble bee species worldwide have undergone declines, driven by a range of factors including habitat loss and fragmentation following agricultural intensification (Goulson et al. 2005, 2008, Carvell et al. 2006a,

Manuscript received 25 March 2015; revised 29 July 2015; accepted 31 July 2015. Corresponding Editor: C. Gratton.

${ }^{5}$ E-mail: johde@ceh.ac.uk
Williams and Osborne 2009, Goulson 2010). As a result, there is great interest in the likely impacts of ongoing modifications to the landscape on ecosystem service delivery by bumble bees, and in methods by which landscapes might be enhanced in terms of suitability for populations of bumble bees and other pollinators (Garibaldi et al. 2014). Such methods include the many agri-environment schemes whose aims include providing foraging resources for pollinating insects (Carvell et al. 2006b, 2007).

As worker bumble bees are central place foragers, the spatial and temporal distribution of resources surrounding the colony is important in determining the energetic returns of foraging trips and ultimately the viability of a colony (Dukas and Edelstein-Keshet 1998). We can 
therefore predict that in order to optimize foraging success, foraging trips might be shorter in both duration (Westphal et al. 2006b) and length (Carvell et al. 2012) where resources within the local landscape are more rewarding. Although it is unlikely that the typical foraging distance alone determines the species-level response to landscape changes, many models of pollinator foraging and pollination services rely on accurate parameterization of foraging distance and resource value of different habitats (Cresswell et al. 2000, Lonsdorf et al. 2009, Raine et al. 2009). Indeed, if land management for bumble bees is to be successful (and cost effective), it is important to have accurate information on how far workers travel to forage and the extent of variation within and between species. There is a growing literature of estimated foraging distances from a range of Bombus species (Osborne et al. 1999, 2008, Walther-Hellwig and Frankl 2000, Knight et al. 2005, Hagen et al. 2011, Carvell et al. 2012, Jha and Kremen 2013), with differences between bumble bee species attributable to interplay between variation in body size (Greenleaf et al. 2007), nest density, colony size, diet breadth, population status, tongue length, and other factors (Knight et al. 2005). However, there are still few studies which analyze wild colonies of several species foraging in a shared landscape (e.g. Chapman et al. 2003, Knight et al. 2005) and quantify within-species variation in relation to landscape characteristics.

Wild colonies of many species of bumble bee are subterranean or concealed in dense vegetation, making them difficult to find (Dramstad 1996). Therefore studies of worker foraging distance have tended to rely upon observations of individual workers from small numbers of wild or experimentally reared colonies (Walther-Hellwig and Frankl 2000, Westphal et al. 2006b, Osborne et al. 2008, Hagen et al. 2011), inferences from worker density and landscape composition (Westphal et al. 2006a, Suzuki et al. 2007), or genetic analyses of bumble bees captured at foraging sites (Chapman et al. 2003, Darvill et al. 2004, Knight et al. 2005, Charman et al. 2010, Dreier et al. 2014b). The first two approaches exhibit various limitations; for example, their methods are too labor-intensive to apply to large samples or must make simplifying assumptions about resource distribution and constancy of foraging ranges. Genetic analyses are also limited in that they cannot provide an exhaustive sample of the underlying population (e.g. Stanley et al. 2013), but in contrast to other approaches, do permit inferences regarding bumble bee spatial ecology based on large numbers of wild colonies. Such studies typically involve sampling worker bees and reconstructing colony memberships on the basis of individual multi-locus genotypes to obtain numbers or densities of colonies represented at sample sites (Herrmann et al. 2007, Knight et al. 2009, Goulson et al. 2010) and infer average foraging ranges for different species (Knight et al. 2005). Recently, these methods have also been used to estimate the average foraging distances of workers from individual colonies
(Carvell et al. 2012, Jha and Kremen 2013). However, previous studies have used data from workers sampled at discrete sites (e.g. spatially separated forage patches or transects), constraining the range of foraging distances and spatial patterns that they are able to detect.

In this study, we used genetic analyses to estimate colony locations and worker foraging distances for five social bumble bee (Bombus) species. These include two species for which little data on worker foraging distances have been published previously (Hagen et al. 2011), namely $B$. ruderatus Fabricius, which is rare in the UK and in decline throughout Europe, and the ecologically similar but more widespread $B$. hortorum. We sampled workers across the entirety of a landscape that varied in habitat composition and combined these data with habitat and floral resources data derived from field surveys and remote sensing, which gave landscape coverage at a fine spatial resolution. This is the first time that this approach has been applied at such a fine spatial scale and to both common and declining species within a shared landscape. Our methods have the advantages of increasing the likelihood of detecting sister workers at multiple sites and of sampling a high proportion of colonies in the landscape (Dreier et al. 2014b). We used our data to test three hypotheses. Firstly, the distance workers travel from the colony to forage varies between species sampled across a common landscape. Secondly, the distances traveled by workers vary between colonies within species. Thirdly, such variation is influenced by the colony's location in the landscape, in terms of habitat composition and landscape structure.

\section{Methods}

\section{Study landscape and study species}

The study was conducted over a $20-\mathrm{km}^{2}$ area of farmland centered on the Hillesden Estate, Buckinghamshire, $\mathrm{UK}\left(51.95^{\circ} \mathrm{N}, 1.00^{\circ} \mathrm{W}\right.$; S1: Fig. S1). The landscape is typical of southern lowland England (UK), being dominated by arable fields of autumn-sown wheat Triticum aestivum L., oilseed rape Brassica napus L., and field beans Vicia faba L., interspersed with fields of permanent pasture (mostly ryegrass Lolium perrene L. and white clover Trifolium repens L.) and isolated small woods. Most fields are bordered by low $(<2 \mathrm{~m})$, shrub hedgerows with scattered, mature trees. The landscape also contains several small villages, giving some cover of gardens and associated suburban vegetation, although this formed a small proportion of the landscape (3.2\%) in comparison to some previous studies (e.g. Osborne et al. 2008, Hagen et al. 2011, Jha and Kremen 2013). The Hillesden Estate itself forms $\sim 10 \mathrm{~km}^{2}$ of the study landscape. This estate has been managed since 2005 under a range of agri-environment options typical of the UK's entry level stewardship (ELS) agri-environment scheme. These include field margins and field corners sown with grass, perennial wildflower and annual bird food seed mixes aimed at promoting a range of farmland 
biodiversity target taxa including pollinating insects (see Redhead et al. [2013] and Broughton et al. [2014] for further details).

Of the five bumble bee species studied, four are common and widespread across much of the UK (B. terrestris L., B. lapidarius L., B. pascuorum Scopoli, B. hortorum L.), while one (B. ruderatus) has suffered significant declines in recent decades and is a conservation priority species listed under Section 41 of the UK Natural Environment and Rural Communities Act 2006. Bombus terrestris and B. lapidarius typically have large colonies and shorttongued workers that visit a wide range of flowers, whereas B. pascuorum and B. hortorum tend to live in smaller colonies and have longer-tongued workers that specialize in foraging at flowers with long corolla tubes (Benton 2006). B. ruderatus is ecologically most similar to $B$. hortorum, these being the longest-tongued UK Bombus species (Prys-Jones and Corbet 1991).

\section{Bumble bee sampling and genetic analysis}

Workers of all five study species were sampled between 20 June and 5 August 2011, using gridded survey maps to ensure full coverage of the entire study landscape (Appendix S1: Fig. S1). Search effort within each $250 \times 250 \mathrm{~m}$ grid cell was broadly proportional to the relative cover of suitable foraging and nesting habitats present. Hence, searches were focused mainly on field boundaries and other non-crop habitat parcels but also included field centers (see Dreier et al. [2014b] for full details). All encountered workers of the target species, whether foraging at flowers or in flight, were caught using a handheld net. Capture locations were recorded using a handheld GPS (Garmin Etrex 10, accurate to $3 \mathrm{~m}$; Garmin, Schaffhausen, Switzerland) and any foraging behaviors noted. The identity of the visited forage plants was also recorded. The tarsal tip was non-lethally removed from the right mid-leg of each bee (Holehouse et al. 2003), preserved in $100 \%$ ethanol until DNA extraction, and the worker released close to its capture location.

DNA was isolated from each tarsal sample using the HotSHOT protocol (Truett et al. 2000). Field identification to species was confirmed with a molecular identification method based on mitochondrial DNA markers to allocate samples to their correct species prior to micro-satellite genotyping (Dreier et al. 2014a,b) for the cryptic species pairs $B$. hortorum/B. ruderatus (Ellis et al. 2006, Stewart et al. 2010) and B. terrestris/B. lucorum L. (H.M.G. Lattorff, personal communication). Individuals were then genotyped at 10-14 microsatellite loci (Dreier et al. 2014a,b). Sister relationships ("sibships") among workers were estimated from individual marker genotypes using the maximum likelihood sibship reconstruction method in COLONY version 2.0 (Wang 2004). Sibship reconstruction was performed independently for each species using a common probability of inference $(0.8)$ as the threshold at which individuals are assigned to a sibship. For full details of the genetic analysis, see Dreier et al. $(2014 a, b)$.

Since body size has been demonstrated to predict foraging range in cross-taxa analyses of pollinators (Greenleaf et al. 2007) we measured intertegular distance (ITD) from six to 10 workers of each of the five bumble bee species collected from either the study landscape or close equivalents (i.e. lowland English arable). All $B$. ruderatus and $B$. hortorum were genotyped to confirm species, as described previously.

\section{Collecting habitat data}

Survey maps of habitat data were based on a land use/land cover (LULC) map derived from two airborne remote sensed sources; Light Detection and Ranging (LiDAR) and hyperspectral imaging. These remote sensed data were acquired by the Natural Environment Research Council Airborne Research and Survey Facility on 28 August 2007. Supervised classification of the hyperspectral dataset, combined with a digital canopy height model derived from LiDAR, produced a high resolution $(0.5 \times 0.5 \mathrm{~m}$ pixels $)$ LULC map. For further details on the collection and processing of the LiDAR and hyperspectral data, see Redhead et al. (2013). For the current study, the LULC map was simplified to nine classes: cropped arable fields, short grass, mixed, nonwoody, seminatural vegetation, garden and urban vegetation, woody vegetation, ELS field margin, road and building, water, and bare soil (see Fig. 1 and Appendix S1: Figure S1), and updated manually to reflect changes in ELS management. The LULC map was used to systematically survey the study landscape in terms of its value for bumble bees. Every mapped LULC polygon representing a discrete habitat parcel (i.e. an area of contiguous land use clearly visible in the field) was surveyed during July and August 2011, to estimate the percentage flower cover (i.e. vegetative cover multiplied by proportion in flower) of target plant species, families, or groups (given in Appendix S1: Table S1). Any changes in the extent of parcels identified in the field were manually added to the LULC map. In total, $18.7 \mathrm{~km}^{2}$ of the study landscape were surveyed in this way. For the remaining $6.5 \%$ of the study area that was not surveyed (because of access restrictions, mostly on pasture fields and suburban areas on the edge of the study area), floral data were estimated by taking the mean cover of values from parcels of the same LULC class within $500 \mathrm{~m}$ of the focal parcel. Handling of the LULC map and survey data, and estimation of colony locations (see following section), was performed in ArcMAP v10.0 (ESRI, Redlands, California, USA).

\section{Estimating colony locations, foraging distances, and relationships with landscape variables}

Locations were estimated for all sibships from which two or more sister workers had been inferred in the sample. Sibships from which only a single worker was 
inferred ("singletons") were excluded from further analyses as they cannot yield a meaningful estimate of colony location (Carvell et al. 2012). Estimated colony locations were derived using a "mean center" approach. This took the mean easting and northing of worker locations from each sibship and plotted the resultant coordinates (Fig. 1). This mean center approach had several advantages over other methods tested in preliminary analyses (Carvell et al. 2012, Dreier et al. 2014b). Other methods were either heavily influenced by outlying worker locations (e.g. centroid of a minimum convex polygon enclosing all workers in a sibship) or drawn to clusters of workers (e.g. median center). The mean center best represents the fact that colonies are more likely to be located within the area of the landscape in which the majority of their workers were found, while avoiding the assumption that colonies are in the immediate vicinity of the largest concentration of workers (Dramstad 1996) or having estimated colony locations biased by the most attractive resource patches. This latter issue is also lessened by the fact that many colonies were represented by widely dispersed workers rather than clusters of workers on single-resource patches (e.g. the colonies shown in Fig. 1). In addition, because the mean center method involved a purely statistical single point output requiring no additional parameters or analysis, no prior assumptions regarding likely foraging distances were required. However, the method still yielded similar estimated colony locations to the kernel density estimation method used previously in the same landscape (Carvell et al. 2012). While individual colony locations estimated using this approach are undoubtedly subject to error, and may still be influenced by greater numbers of workers at attractive flower patches, this is not likely to have been systematic across all colonies within a species. To test the validity of our approach to estimating colony locations, and the potential effects of sibship size on these estimates, we randomly resampled combinations of two, three, and four workers from each sibship from which sufficient workers were sampled and estimated colony location from each resample. We then examined the effect on estimated colony location and foraging distances.

Mean center locations were "snapped" (i.e., moved to coincide exactly with the coordinates of another feature) to the nearest LULC class that might have formed

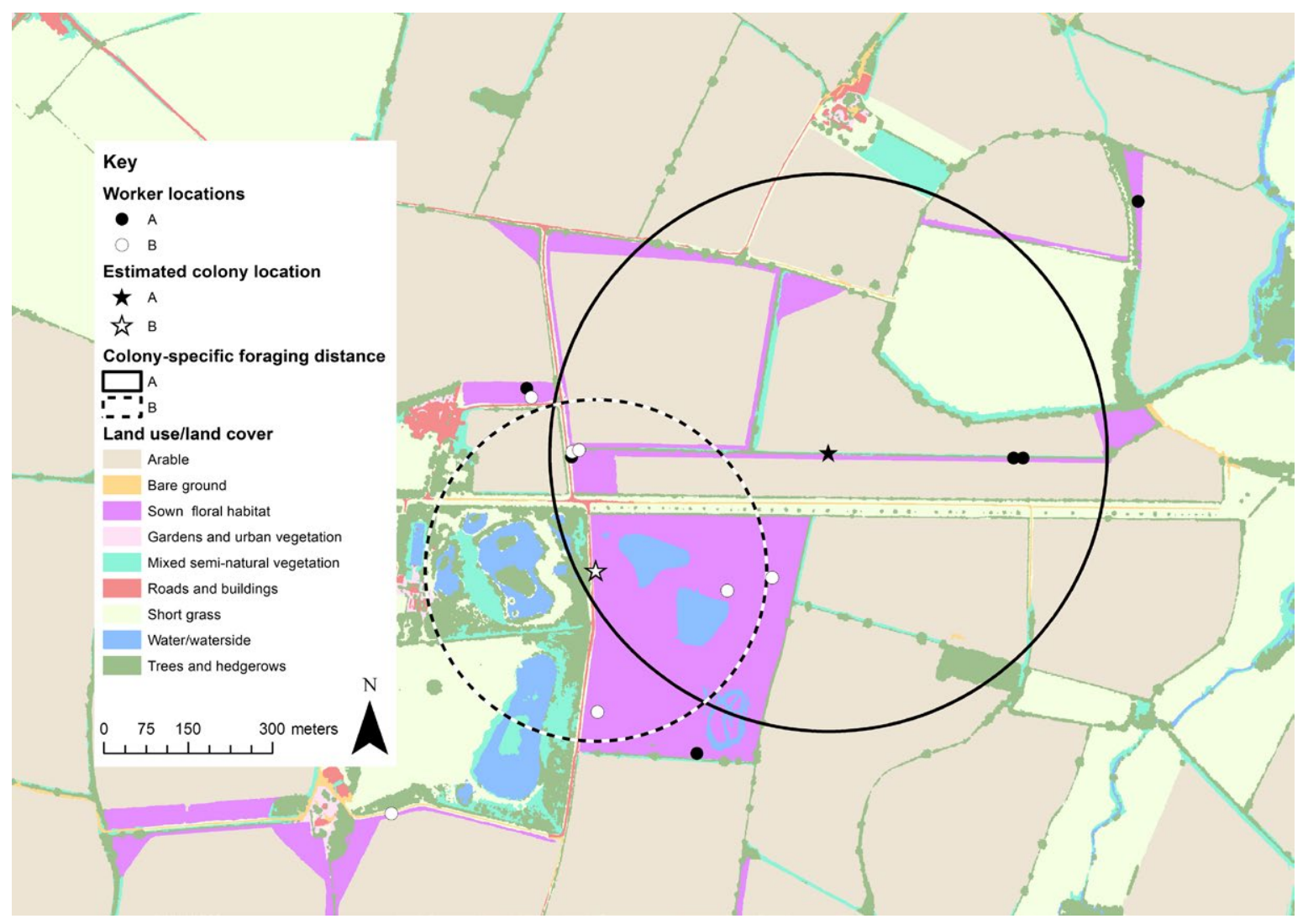

FIg. 1. Example of the colony location estimation method, overlaid on the land use/land cover map, for two bumble bee colonies (A and B). Black/white circular symbols = capture locations of workers determined to be from a given colony following genetic analysis. Stars represent mean centers of these locations, i.e., estimated colony locations. Solid/dashed lines represent buffers with a radius equal to the mean distance of all full sister workers to their estimated respective colony locations (i.e., colony-specific foraging distance). 
suitable nesting habitat for bumble bees (i.e. all classes except cropped arable fields, roads, buildings and water; Benton 2006). The straight-line distance of each worker from its capture location to estimated colony location was calculated as a measure of worker foraging distance. The mean of these distances for all workers in a sibship was then calculated to give a colony-specific foraging distance. Most locations did not require snapping, and, for those that did, the snapping process made a mean difference to colony-specific foraging distances of only $4.5 \mathrm{~m}$ across all species.

To estimate the resource quality of the landscape surrounding each colony, a buffer with a radius equal to its colony-specific foraging distance was created around the colony location (Fig. 1). The proportion of each LULC class and the floral cover of plant groups within this buffer were then determined. Mixed, non-woody, seminatural vegetation, and ELS margins were combined to a single mixed seminatural vegetation class. This comprised the bulk of non-woody seminatural habitats including sown and non-sown field margins, roadside verges, vegetated waste ground, and fallow arable land. We analyzed only those LULC classes which formed a major component of the study landscape and were roughly evenly distributed across it. These were arable, combined mixed seminatural vegetation, short grass, and woody vegetation. Floral cover of surveyed plant groups was further grouped into more specific subsets in terms of the plants' relative value as forage resources for bumble bees. These groupings were noncrop (all floral cover not attributable to oilseed rape or field beans), visited (all floral cover attributable to plant groups visited by foraging workers during sampling), and preferred (all floral cover attributable to the five plant groups with the highest mean number of observed worker visits to species within the group, as listed in Appendix S1: Table S1). A variety of landscape structure metrics with the potential to provide ecologically informative measures of the spatial configuration of habitats or influence the foraging distances of bumble bees were trialed in preliminary investigations. Three of these, that were not significantly intercorrelated, were selected to reflect key aspects of landscape structure in the context and at the scale of our study landscape:

(1) Mean patch edge:area ratio for patches of mixed seminatural vegetation; a measure of the fragmentation of resource patches surrounding each colony, incorporating patch size (Riitters et al. 1995, Moser et al. 2002).

(2) Mean shape index for patches of mixed seminatural vegetation, calculated as patch perimeter divided by the square root of patch area, multiplied by 0.25 ; a measure of the average complexity of patch shapes (equaling one for perfectly square patches, decreasing without limit as patches become more irregular), independent of patch area (Riitters et al. 1995, Moser et al. 2002).
(3) Hedgerow proximity index, calculated by summing the distance to the nearest three hedgerow intersections; an index of the amount and complexity of hedgerow in the local landscape (Cranmer et al. 2012).

\section{Statistical analyses}

The relationship between each habitat variable and $\log$ (base 10) transformed colony-specific foraging distance was analyzed for each species by independent general linear models (GLMs) performed in R (R Core Team 2013). Conformity to the assumptions of GLM (homogeneity of variance, distribution of residuals) was checked by plotting in R. Colonies with less than 95\% coverage of habitat data within the buffer were excluded from these analyses $(n=21)$. Colonies with a mean colony-specific foraging distance of less than $20 \mathrm{~m}$ were also excluded $(n=24)$. The latter were excluded because such colonies were likely to have resulted from sampling related workers in a single resource patch. These two exclusions applied approximately equally across species and resulted in only $8 \%$ of colonies being excluded from further analyses. Because some of the variables analyzed were to some degree correlated (e.g. proportional land cover types, subsets of floral cover of surveyed plant groups) all GLMs were conducted independently on single variables, and the explanatory power of each variable assessed by examining $R^{2}$ and AIC values to identify the best-fitting models (Burnham and Anderson 2002).

\section{RESULTS}

A total of 2577 workers were genotyped from the five target species (sample sizes given in Table 1). The total estimated number of colonies within the landscape varied between species (Table 1), but not in direct proportion to the number of individual workers sampled, with some species having higher proportions of singletons (e.g. B. hortorum and B. terrestris). Recaptures (as determined from multi-locus genotypes) were infrequent (12 workers).

Estimated worker foraging distances differed significantly between species (i.e. $P<0.05$ ) (Table 1 , one-way ANOVA, $\left.F_{4,1551}=26.42, P<0.01\right)$. Species mean foraging distances formed two groups (Tukey post hoc tests, Appendix S1: Table S1) with shorter distances of $\sim 300 \mathrm{~m}$ for $B$. pascuorum and $B$. hortorum and significantly longer distances of $\sim 500 \mathrm{~m}$ for B. terrestris, B. lapidarius, and $B$. ruderatus. Maximum foraging distances were considerably greater, with an individual worker of $B$. terrestris reaching $2878 \mathrm{~m}$ from its estimated colony location (see Table 1 for other species maxima).

Colony locations estimated using only two workers resampled from sibships with more than two workers were $244 \pm 4.33 \mathrm{~m}$ (mean \pm standard error) from locations estimated using all workers in a sibship (Appendix S1: Fig. S1 A), but this resulted in a mean decrease of 
TABLE 1. Sample sizes $(N)$ and descriptive statistics for worker foraging distances, along with intertegular distance (ITD), for each of the five Bombus species.

\begin{tabular}{|c|c|c|c|c|c|c|c|c|}
\hline Species & ITD & $\begin{array}{c}\text { All } \\
\text { workers }\end{array}$ & $\begin{array}{l}\text { Non- } \\
\text { singletons }\end{array}$ & $\begin{array}{c}\text { All } \\
\text { sibships }\end{array}$ & $\begin{array}{l}\text { Non-singleton } \\
\text { colonies }\end{array}$ & $\begin{array}{l}\text { Mean (range) } \\
\text { workers per } \\
\text { colony }\end{array}$ & $\begin{array}{l}\text { Mean worker } \\
\text { foraging } \\
\text { distance }\end{array}$ & $\begin{array}{l}\text { Maximum } \\
\text { worker foraging } \\
\text { distance }\end{array}$ \\
\hline B. terrestris & $5.56 \pm 0.14$ & 382 & 187 & 264 & 69 & $2.71(2-8)$ & $551 \pm 39.83$ & 2878 \\
\hline B. lapidarius & $4.54 \pm 0.13$ & 1171 & 774 & 668 & 271 & $2.86(2-11)$ & $536 \pm 16.02$ & 2059 \\
\hline B. pascuorum & $4.37 \pm 0.11$ & 548 & 311 & 360 & 123 & $2.53(2-7)$ & $337 \pm 19.92$ & 1808 \\
\hline B. hortorum & $4.84 \pm 0.24$ & 262 & 117 & 193 & 48 & $2.44(2-6)$ & $273 \pm 20.15$ & 810 \\
\hline B. ruderatus & $5.58 \pm 0.02$ & 214 & 168 & 88 & 42 & $4.00(2-19)$ & $502 \pm 33.71)$ & 2350 \\
\hline
\end{tabular}

Notes: Sample sizes are given with and without "singletons" (colonies from which only a single worker was sampled). All distances quoted are in meters. Mean workers per colony is shown for non-singleton colonies only. ITD and foraging distance are shown \pm standard error.

only $64 \pm 10.51 \mathrm{~m}$ in estimated colony-specific foraging distance (Appendix S1: Fig. S1 B). Therefore while colonies from which fewer workers were sampled yielded different colony locations, the effect of sibship size on estimated colony-specific foraging distance, and on species mean foraging distances as presented in Table 1, is comparatively minor and largely consistent across species (Appendix S1: Fig. S1 C and Table S1).

Colony-specific foraging distances varied widely between colonies of the same species (Table 1) and a range of habitat variables showed significant relationships with colony-specific foraging distances across species (Table 2). Overall there was a strong, significant negative effect of cover of mixed seminatural vegetation, such that increasing cover decreased the colony-specific foraging distances of all species (Fig. 2). This relationship was markedly weaker for B. terrestris. Cover of arable land showed the reverse relationship (Fig. 2), such that greater arable cover resulted in greater colonyspecific foraging distances. This relationship was strongest for B. terrestris and B. lapidarius.

Significant effects of floral cover on colony-specific foraging distances were found only for non-crop vegetation. A significant, negative relationship between colony-specific foraging distance and non-crop floral cover surrounding the colony was observed for all species, with highest model fit for longer-tongued species (B. pascuorum, B. hortorum, and $B$. ruderatus). Limiting the floral cover data to workervisited plant groups made little difference to model fit. However, further refinement to worker-preferred plant groups improved the explanatory power of the models (Table 2), especially for the two long-tongued species $B$. hortorum and B. ruderatus. The decline in colony-specific foraging distance with increased worker-preferred floral cover was also notably steeper for these two species (Fig. 3).

Among the landscape structure metrics, there was a significant positive relationship between colony-specific foraging distance and mean edge:area ratio for all species (Table 2). For B. terrestris, B. lapidarius, and B. ruderatus, mean edge:area ratio showed lower AIC than proportion of mixed seminatural vegetation. For $B$. hortorum and B. pascuorum, the opposite was true, with mean edge:area ratio of secondary importance compared to proportion of mixed seminatural vegetation. In contrast, shape index only had a significant effect in B. hortorum and B. pascuorum, and in neither case did it improve model fit above total cover of mixed seminatural vegetation. The hedgerow proximity index showed low model support for all species (Table 2).

\section{Discussion}

In this study we used genetic analyses to determine colony membership for worker bumble bees of five species sampled across an agricultural landscape and thereby estimate colony locations and foraging distances at the level of individual workers. The results confirmed all three of our hypotheses. Firstly, we found significant differences in worker foraging distances between the five study species, which could be divided into longer(B. lapidarius, B. terrestris, and B. ruderatus) and shorter- (B. hortorum, B. pascuorum) range foragers. Secondly, we showed that at the level of individual colonies, foraging distance varied widely within each species. Thirdly, our results confirm that this variation is dependent on the location of colonies within the landscape with respect to the availability and configuration of floral resources. It is possible that the estimated location and mean foraging distance of each colony may be subject to some error associated with the number of workers sampled from the colony. However, our resampling of pairs of sister workers demonstrates that there is no evidence to suggest a systematic bias which would affect comparisons between species or relationships with habitat. In addition, because of the landscape structure and configuration of the study area, small shifts in the exact estimate of colony location do not result in significant differences in the landscape metrics measured. While our estimates cannot be considered an absolute measure of the foraging distances of each sampled colony, they provide a basis for comparative assessment of responses to landscape and habitat variables among different species, since the same fine-scale sampling and modeling approaches were applied to all species. Our results confirm 
TABLE 2. Results of linear regression of colony-specific foraging distance against log-transformed habitat variables, for five Bombus species.

\begin{tabular}{|c|c|c|c|c|c|c|c|c|c|}
\hline \multirow[b]{2}{*}{ Species } & \multicolumn{2}{|c|}{ LULC } & \multicolumn{4}{|c|}{ Floral cover } & \multicolumn{3}{|c|}{ Landscape structure metrics } \\
\hline & $\mathrm{AR}$ & MV & ALL & $\mathrm{NC}$ & WV & WP & EA & SI & HI \\
\hline \multicolumn{10}{|c|}{ B. terrestris $(N=65, \mathrm{df}=63)$} \\
\hline Slope & 2.713 & -2.096 & 0.420 & -4.020 & -4.092 & -4.928 & 1.383 & 0.151 & 0.072 \\
\hline$R^{2}$ & 0.327 & 0.072 & -0.013 & 0.203 & 0.206 & 0.219 & 0.146 & -0.016 & -0.015 \\
\hline$P$ & $<0.001 * * *$ & $0.017^{*}$ & 0.652 & $<0.001 * * *$ & $<0.001 * * *$ & $<0.001 * * *$ & $0.001 * * *$ & 0.891 & 0.831 \\
\hline AIC & 64.045 & 84.974 & 90.643 & 75.083 & 74.815 & 73.753 & 79.578 & 90.835 & 90.808 \\
\hline \multicolumn{10}{|c|}{ B. lapidarius $(N=248, \mathrm{df}=246)$} \\
\hline Slope & 1.955 & -3.469 & 0.061 & -2.686 & -2.581 & -4.002 & 1.574 & -0.450 & 0.520 \\
\hline$R^{2}$ & 0.177 & 0.153 & -0.004 & 0.063 & 0.058 & 0.085 & 0.189 & -0.002 & 0.035 \\
\hline$P$ & $<0.001 * * *$ & $<0.001 * * *$ & 0.873 & $<0.001 * * *$ & $<0.001 * * *$ & $<0.001 * * *$ & $<0.001 * * *$ & 0.474 & $0.002 * *$ \\
\hline $\mathrm{AIC}$ & 216.108 & 223.190 & 265.328 & 248.298 & 249.448 & 242.182 & 212.363 & 264.835 & 255.401 \\
\hline \multicolumn{10}{|c|}{ B. pascuorum $(N=108, \mathrm{df}=106)$} \\
\hline Slope & 3.396 & -4.616 & -0.930 & -6.359 & -6.341 & -9.082 & 2.636 & -3.601 & 0.754 \\
\hline$R^{2}$ & 0.354 & 0.481 & 0.009 & 0.416 & 0.417 & 0.428 & 0.315 & 0.138 & 0.057 \\
\hline$P$ & $<0.001 * * *$ & $<0.001 * * *$ & 0.165 & $<0.001 * * *$ & $<0.001 * * *$ & $<0.001 * * *$ & $<0.001 * * *$ & $<0.001 * * *$ & $0.008 * *$ \\
\hline $\mathrm{AIC}$ & 109.362 & 85.621 & 155.535 & 98.483 & 98.227 & 96.128 & 113.511 & 138.127 & 147.682 \\
\hline \multicolumn{10}{|c|}{ B. hortorum $(N=44, \mathrm{df}=42)$} \\
\hline Slope & 2.059 & -4.411 & -0.013 & -4.294 & -4.325 & -7.339 & 1.252 & -5.982 & -0.368 \\
\hline$R^{2}$ & 0.165 & 0.507 & -0.024 & 0.253 & 0.256 & 0.383 & 0.084 & 0.205 & 0.001 \\
\hline$P$ & $0.004 * *$ & $<0.001 * * *$ & 0.986 & $<0.001 * * *$ & $<0.001 * * *$ & $<0.001 * * *$ & $0.032^{*}$ & $0.001 * *$ & 0.312 \\
\hline AIC & 38.510 & 15.321 & 47.485 & 33.641 & 33.438 & 25.225 & 42.596 & 36.376 & 46.402 \\
\hline \multicolumn{10}{|c|}{ B. ruderatus $(N=41, \mathrm{df}=39)$} \\
\hline Slope & 1.553 & -3.034 & -0.066 & -4.849 & -4.880 & -13.590 & 2.661 & -1.368 & 0.093 \\
\hline$R^{2}$ & 0.143 & 0.373 & -0.025 & 0.364 & 0.369 & 0.508 & 0.485 & -0.007 & -0.023 \\
\hline$P$ & $0.009 * *$ & $<0.001 * * *$ & 0.938 & $<0.001 * * *$ & $<0.001 * * *$ & $<0.001 * * *$ & $<0.001 * * *$ & 0.401 & 0.764 \\
\hline AIC & 18.607 & 5.788 & 25.968 & 6.360 & 6.086 & -4.130 & -2.294 & 25.224 & 25.878 \\
\hline
\end{tabular}

Notes: $N$ refers to number of colonies. For land-use/land-cover (LULC) classes, results are shown for arable (AR) and mixed seminatural vegetation (MV). Cover of short grass and woody vegetation were nonsignificant across all species and are not reported. Floral cover variables are: total for all plant groups (ALL), non-crop (NC), worker-visited (WV), and worker-preferred (WP) species or groups. Landscape structure metrics are: mean edge:area ratio (EA), mean shape index (SI), and hedgerow proximity index (HI).

Significance levels are indicated by $* P<0.05 ; * * P<0.01 ; * * * P<0.001$.

the potential for bumble bees to show significant foraging plasticity in response to changes in resource availability (Jha and Kremen 2013), but suggest that differences between species and the scale of land-use changes could be critical in designing management practices to conserve bee populations and enhance pollination services.

\section{Variation in worker foraging distances between species}

Our results confirm those of previous studies that suggest that species show significant differences in foraging range (Walther-Hellwig and Frankl 2000, Darvill et al. 2004, Knight et al. 2005), and that there is a degree of consistency between average foraging ranges within species across broadly similar landscape types (Knight et al. 2005). Estimates of mean and maximum foraging distance for each species (Table 1) fell within the range of previous estimates for B. terrestris, B. lapidarius, and B. pascuorum. For example, we found that $B$. terrestris workers may forage several kilometers from the colony (Walther-Hellwig and Frankl 2000, Knight et al. 2005, Westphal et al. 2006a, Osborne et al. 2008,
Hagen et al. 2011, Wood et al. 2015) and that B. pascuorum workers generally travel shorter distances (Darvill et al. 2004, Knight et al. 2005, Wood et al. 2015), although occasional individuals can still be found almost $2 \mathrm{~km}$ from the colony (Carvell et al. 2012). While some studies have suggested that B. lapidarius has a similar mean foraging range to $B$. pascuorum (Knight et al. 2005, Carvell et al. 2012), in our study, $B$. lapidarius was more similar in its foraging range to B. terrestris (Walther-Hellwig and Frankl 2000, Westphal et al. 2006a, Wood et al. 2015). This finding might be expected from the similar ecology and population status of the two species. However, it should be borne in mind that the extent of variation seen within species in this study, even in a single landscape, renders it unsurprising that studies conducted in different landscapes or with different sampling approaches find different results for the same species.

Very few previously published foraging distances are available for workers of B. hortorum (Wood et al. 2015) or B. ruderatus (Hagen et al. 2011). Both are long-tongued species, with a high level of specialization on long-corolla 

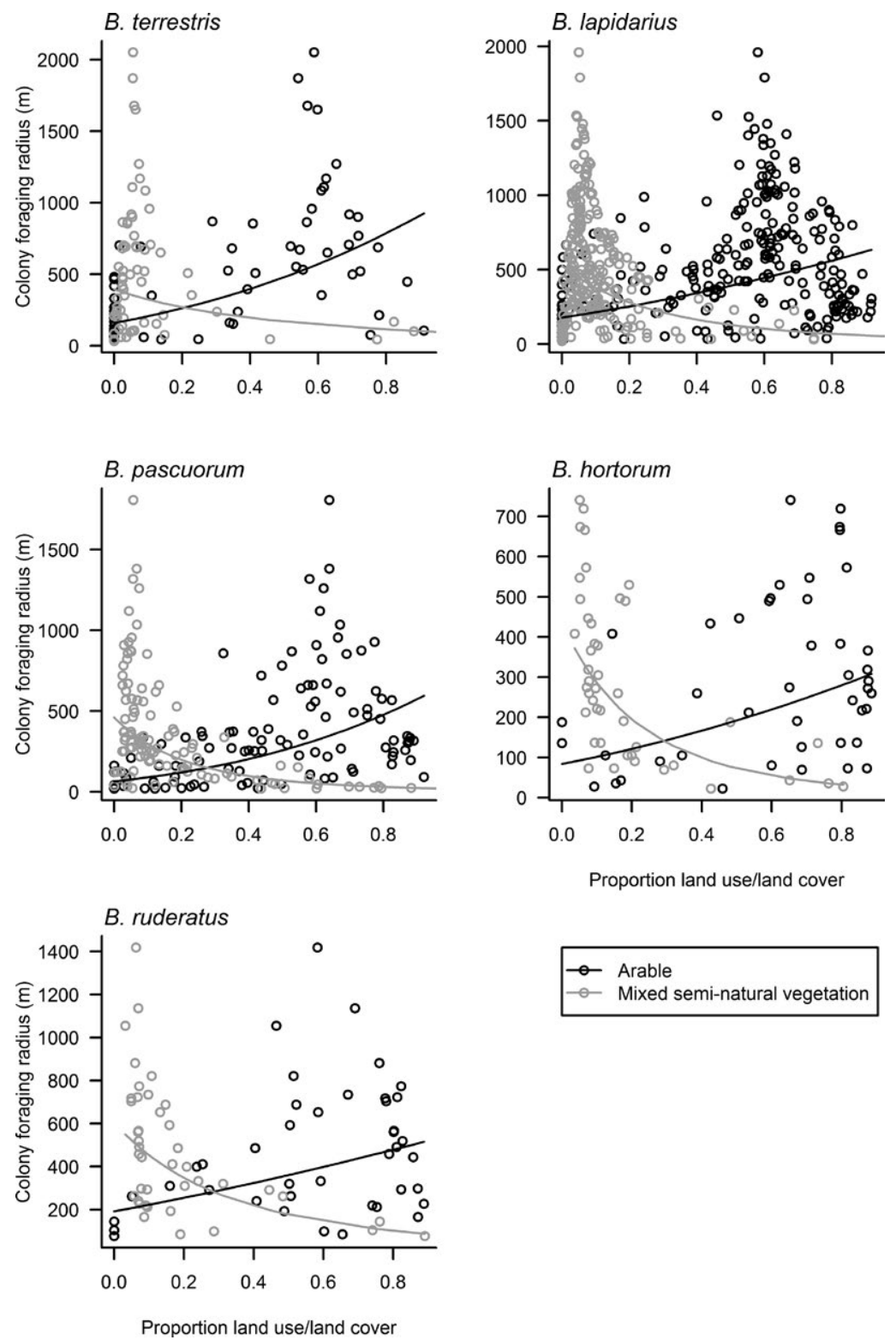

FIG. 2. Plots of proportional cover of arable fields (black lines and symbols) and mixed seminatural vegetation (gray lines and symbols) against colony-specific foraging distance for five Bombus species. Fitted lines are back-transformed from independent linear regressions of log-transformed data (statistics in Table 2).

flowers such as red clover, Trifolium pratense L. (Carvell et al. 2006b), lending them particular ecological importance as pollinators (Garratt et al. 2014). However, the two species have shown contrasting population trends, with $B$. hortorum remaining widespread throughout Europe (Goulson et al. 2005) and B. ruderatus showing significant contractions in its native range. Our results showed B. hortorum to have the shortest mean and maximum worker foraging distances of the five species, whereas the values for $B$. ruderatus were relatively high, as previously found from radio tracking of a single $B$. ruderatus worker by Hagen et al. (2011). This is counter to the expectation that species with the shortest foraging ranges should be most at risk from lack of forage in the local landscape, and thus most threatened by changes in land use. It is therefore unlikely that typical foraging distance alone determines the species-level response to landscape changes, especially given the evident plasticity in foraging range seen in this study.

Identifying what determines the observed foraging range of a species is complex. Factors such as body size have been demonstrated to predict foraging range across broad taxonomic species groups (Greenleaf et al. 2007). However, the data used by Greenleaf et al. (2007) and 

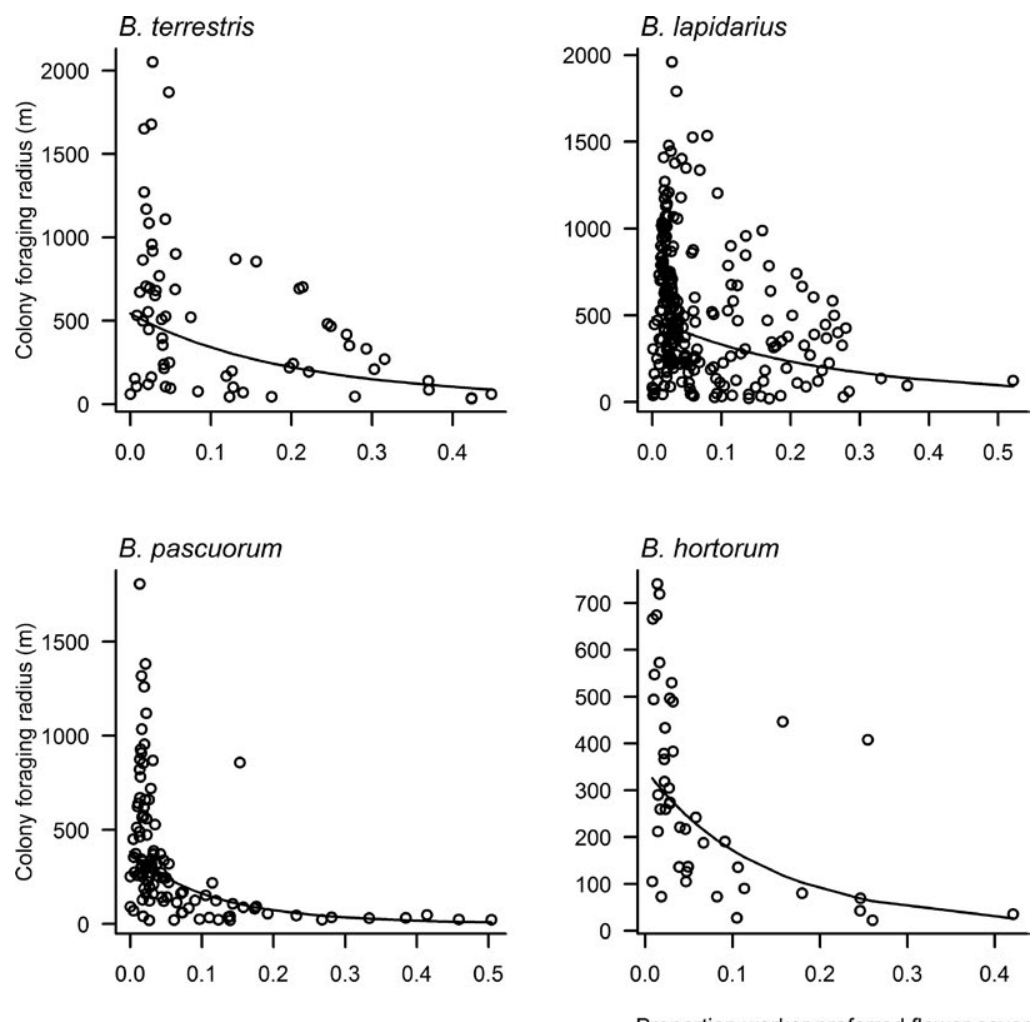

B. ruderatus

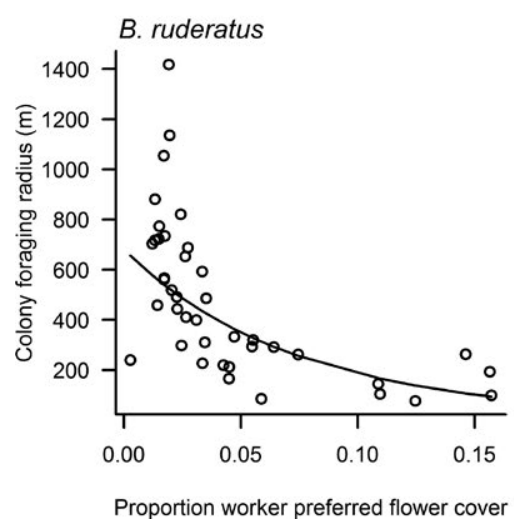

Proportion worker preferred flower cover

FIG. 3. Plots of proportional cover of worker-preferred floral groups (specified in Appendix S1: Table S1) against colonyspecific foraging distance for five Bombus species. Fitted lines are back-transformed from independent linear regressions of log-transformed data (statistics in Table 2).

the results of other studies (Darvill et al. 2004, Knight et al. 2005) suggest that body size does not predict foraging range across bumble bee species in particular. Similarly, our longer- and shorter-range forager groups do not correspond clearly to differences in ITD (Table 1), tongue length, diet breadth, colony size, or population trends. This, and the evident variation in foraging distance within species, suggests that observed foraging range is determined by an interplay of factors associated with the physiological capabilities of the bumble bee, the requirements of the colony and the availability and configuration of suitable resources (depending on a species physiology and behavior). Thus it remains to be seen whether the groupings observed here are generalizable outside of this study.

\section{Relationships between habitat composition and colony-specific foraging distances}

The amount of floral resources provided by non-crop vegetation, whether measured directly or by proxy (as cover of the mixed seminatural vegetation land cover class), always showed a significant negative relationship with foraging distance, such that colonies in areas of the 
landscape with the least floral resources had, on average, workers foraging further from the colony. Longer foraging distances may be either beneficial or injurious at the colony level, since workers face a trade-off between the increased costs of foraging and potential energetic gains from access to additional resources (Schmid-Hempel and Schmid-Hempel 1998, Cresswell et al. 2000). Although relationships between habitat and foraging distance should be interpreted with caution, due to potential influences from variables not measurable by the methods of this study (e.g., differing colony size, population density, intensity of competition), our results suggest that contrasting situations may occur in different species.

Widespread species with longer foraging distances, such as $B$. terrestris and B. lapidarius, may be more flexible in their ability to compensate for a resource-poor local landscape by increasing search effort to find more distant patches of high-quality forage (Walther-Hellwig and Frankl 2000, Westphal et al. 2006a, Osborne et al. 2008). Both are short-tongued, generalist species, which can also gain access to longer-corolla flowers via behaviors such as nectar robbing (Stout et al. 2000). These characteristics mean that these species have the widest range of potential food sources, and so are most likely both to find beneficial resources in the local landscape and, where these are lacking, to obtain access to such resources by flying further. This is supported in our study by the fact that $B$. terrestris and $B$. lapidarius show a stronger relationship with cover of arable land than with any subset of mixed seminatural vegetation, suggesting that a wide range of habitats provide forage. In contrast, $B$. ruderatus was the only species of the longer foraging range group to retain strong relationships between colony-specific foraging distance and floral cover. The relationship with foraging distance for B. ruderatus was especially strong with worker-preferred floral cover, reflecting the specialization of this bumble bee species on a small subset of forage plants. Only 13 plant species were observed as forage plants for B. ruderatus workers captured while foraging, with red clover accounting for $72 \%$ of these workers, in comparison to 25-32 plant species observed as forage plants for other Bombus species. Because of this, B. ruderatus workers from colonies in resource-poor parts of the landscape must travel long distances to reach suitable forage patches. They may not, however, be able to reach a point where the proportional cover of resources offsets the costs of increased travel. Similar situations may hold for other rare or declining species such as B. distinguendus Morawitz, the only other rare UK bumble bee species for which foraging distance has been directly studied. This species has been shown to have a foraging distance similar to our result for B. ruderatus $(391 \mathrm{~m})$, and a similar level of specialization on floral resources which are increasingly less common under agricultural intensification (Charman et al. 2010).

Neither B. pascuorum nor B. hortorum are showing the declines that might be expected given their comparatively short average foraging distances and strong relationships between foraging distance and local habitat, although there is evidence that their prevalence in the bumble bee community has declined in modern arable landscapes (Bommarco et al. 2011). Bombus pascuorum has a medium tongue length and has been associated with a wide range of forage plants (Dramstad and Fry 1995), including flowering crops (Herrmann et al. 2007, Garratt et al. 2014). Although we did not directly test specialization of individual bumble bee species, we infer from the small increase in model fit from non-crop to worker visited and worker-preferred floral cover that $B$. pascuorum derives some benefits from species other than those on which we observed it to forage. This may allow it to maximize the value of the local area by intensive use of all available resources, as suggested for B. muscorum L. by Walther-Hellwig and Frank1 (2000). The widespread status of $B$. hortorum despite its similarity to the declining $B$. ruderatus has been a continuing enigma, with suggested explanations including differences between the species in their proximity to the edges of their global distributions (Goulson et al. 2005) and, as recent evidence from the current study landscape suggests, lower colony densities and levels of genetic diversity in B. ruderatus (Dreier et al. 2014b). Our results add to these findings by suggesting that $B$. ruderatus uses the landscape at a different spatial scale, more similar to that of B. lapidarius and B. terrestris, despite an apparent preference for a restricted subset of plant groups where they occur.

For all species, total floral cover including cover of flowering crops did not show a significant effect on colony-specific foraging distance, despite the presence of flowering field bean and some fields of late-flowering oilseed rape. A similar result was found for B. vosnesenskii (Radoszkowski) in the USA by Jha and Kremen (2013), with no apparent effect of total floral cover, although there are considerable differences in spatial scale and sampling approach between the study of Jha and Kremen (2013) and the current study. The most abundant flowering crop in our landscape (and in the UK), oilseed rape, has been implicated in affecting bumble bee colony size, local worker abundance and worker foraging patterns, but these effects can be short-lived, due to its comparatively short flowering period (Westphal et al. 2003, 2009, Kovács-Hostyánszki et al. 2013, Persson and Smith 2013). In the present study, surveys were conducted well after the peak flowering period of oilseed rape so that even later-flowering fields are likely to have already declined in value. Indeed no workers were observed foraging on oilseed rape in our study, whereas some previous studies have observed certain species to forage almost exclusively on this resource at peak flowering times (Stanley et al. 2013). The lack of response to mass-flowering crops in this study emphasizes the importance of longer-flowering seminatural resources for sustaining the full colony cycle of bumble bees. It should also be noted that mass flowering crops 
are not the only floral resource to show strong variation over the course of the year. Many of the seminatural habitats in the study landscape are likely to differ in their provision of floral resources over the course of the bumble bee colony life cycle. Because of this, colonies may be established in parts of the landscape which subsequently become resource poor or parts of the landscape which are resource rich for foraging workers may be inhospitable to colony establishment because of a lack of floral resources earlier in the year (Goulson 2010).

\section{Relationships between landscape structure and colony-specific foraging distances}

The spatial arrangement of resources is well established as a potential driver of pollinator abundance and foraging patterns (Rundlof et al. 2008, Cranmer et al. 2012), and, at larger scales, habitat fragmentation is frequently cited as a major driver of biodiversity loss (Krauss et al. 2010). At the scale of the current study, it was evident that landscape structure was important to varying degrees for the different species of bumble bee.

In agricultural landscapes dominated by large open spaces, linear features such as hedgerows may provide important flyways for pollinators that facilitate movement between forage patches (Cranmer et al. 2012). Our study found only weakly supported relationships between the abundance and proximity of hedgerows in the local landscape and worker foraging distances. This does not mean that hedgerows are not important to worker movements but rather that, in our landscape, hedgerows did not promote a significant increase in the mean distance traveled. As hedgerows are commonplace across the study landscape (Appendix S1: Fig. S1), it is unlikely that local hedgerow proximity is a major factor in determining the accessibility of floral resources, and is thus relatively uninfluential compared to the abundance of the resources themselves.

Species with longer foraging distances responded more strongly to edge:area ratio than to total cover of mixed seminatural vegetation. In the study landscape, edge:area ratio was largely driven by whether the local landscape contained many small patches or few large ones, with a comparatively weak influence of patch shape, thus giving a measure of resource fragmentation. For the three longer-range species, models with edge:area ratio showed a better fit than total resource area alone, suggesting that workers of these species travel further not only when resources are scarce, but when they are patchy and scattered. Bombus ruderatus showed the strongest relationship with this variable, corroborating previous suggestions that $B$. ruderatus requires not only the presence of long corolla flowers but large, continuous tracts of habitat containing these species (Goulson et al. 2005).

Over longer foraging distances, travel between patches becomes more feasible, as does covering an elongated or irregular patch, so it might be expected that total area and fragmentation are more important than the shape of patches for species foraging over greater distances. Indeed, B. terrestris, B. lapidarius, and B. ruderatus did not show any significant relationship with patch shape index. Although B. hortorum and B. pascuorum did show a significant relationship, patch shape index explained less variation than total mixed seminatural vegetation cover, suggesting that even for shorter-range foragers, the shape of individual patches is of lesser importance than the total area of available forage. A larger total area of floral resource, in large patches, spaced within the mean foraging range of the species, remains the most beneficial situation for all five species. Under current UK agri-environmental practice, many options targeted at pollinators are implemented as concentrated field corners or linear field margins. Although the latter may have a high edge:area ratio compared to the former, they are likely to remain beneficial if they are of sufficient total area and placed in such a way as to minimize the distance between patches in the landscape.

\section{Implications for design and implementation of land management for bumble bee conservation}

Overall our study suggests that even within a relatively small landscape area, bumble bee worker foraging distances vary according to resource availability as determined by landscape context. Several studies have asserted that common bumble bees may form useful proxies for rare, and thus more difficult to study, species by virtue of shared ecological attributes such as body size, nesting ecology, tongue length, or life cycle (Walther-Hellwig and Frank1 2000, Greenleaf et al. 2007, Jha and Kremen 2013). By sampling both common and rare species within a shared landscape, our study confirms that even ecologically and morphologically similar species can respond to landscape composition and structure in very different ways (Darvill et al. 2004, Knight et al. 2005, Stanley et al. 2013). Despite the variation shown within and between species, our results suggest that management actions involving provision of specific floral resources, for example by sowing of targeted wildflower mixtures on non-cropped areas under agri-environment schemes (Carvell et al. 2007), are likely to reduce the distance workers are required to travel in order to forage, for many bumble bee species. These effects are likely to be most pronounced where resources are arranged in such a way as to increase connectivity at a scale relevant to the foraging range of most colonies.

We used the modeled relationships with mixed vegetation and preferred floral cover (Figs. 2 and 3), to estimate the proportional cover of forage plants at which workers of the five species would forage at or below their species mean forage distance. Our estimates suggest that, in the typical UK lowland arable landscape studied here, this could be achieved at 5-10\% cover of non-crop, seminatural vegetation or $1-3 \%$ 
floral cover of preferred forage plants. In practice this equates to 3-6 ha (non-crop vegetation) and 1-1.8 ha (preferred forage plants) within a 60-ha area (an area with a radius, $437 \mathrm{~m}$, that is the approximate mean foraging distance across all species). While provisioning workers at the time of peak foraging activity is likely to be an important contributor to the survival and productivity of bumble bee colonies, there remain other potential targets for conservation management. Further work on the impact of the landscape on colony establishment, survival, and queen dispersal would be valuable in quantifying the importance of forage at different times of year, and in identifying requirements for nesting and overwintering sites.

\section{ACKNOWLedgments}

We are extremely grateful to the CEH field survey team, coordinated by Sarah Hulmes, for sampling bumble bees and conducting habitat surveys (Lucy Hulmes, Jodey Peyton, Jo Savage, Sam Amy, Roselle Hyman, Gemma Baron and Rachel MacDonald). We thank Robin Faccenda and Richard Franklin of Faccenda Farms, and other landowners, for kind permission to work on the Hillesden Estate and surroundings; Hannah Dean for data management; H. Michael Lattorff for use of his primers for the molecular discrimination of B. terrestris and B. lucorum workers and Ian Warren for assistance with laboratory work. This research was supported by the Insect Pollinators Initiative (grant BB/I000925/1). The Insect Pollinators Initiative is funded jointly by the Biotechnology and Biological Sciences Research Council, the Department for Environment, Food and Rural Affairs, the Natural Environment Research Council, The Scottish Government and The Wellcome Trust, under the Living with Environmental Change Partnership. Acquisition of remote sensing data was funded by Syngenta Plc.

\section{Literature Cited}

Benton, T. 2006. Bumble bees: the natural history \& identification of the species found in Britain. Collins, London.

Bommarco, R., O. Lundin, H. G. Smith, and M. Rundlöf. 2011. Drastic historic shifts in bumble-bee community composition in Sweden. Proceedings of the Royal Society B: Biological Sciences 279:309-315.

Broughton, R. K., R. F. Shore, M. S. Heard, S. R. Amy, W. R. Meek, J. W. Redhead, A. Turk, and R. F. Pywell. 2014. Agri-environment scheme enhances small mammal diversity and abundance at the farm-scale. Agriculture, Ecosystems \& Environment 192:122-129.

Burnham, K. P., and D. R. Anderson. 2002. Model Selection and Multimodel Inference: A Practical Information-Theoretic Approach. Second edition. Springer-Verlag, New York.

Carvell, C., D. B. Roy, S. M. Smart, R. F. Pywell, C. D. Preston, and D. Goulson. 2006a. Declines in forage availability for bumble bees at a national scale. Biological Conservation 132:481-489.

Carvell, C., P. Westrich, W. R. Meek, R. F. Pywell, and M. Nowakowski. 2006b. Assessing the value of annual and perennial forage mixtures for bumble bees by direct observation and pollen analysis. Apidologie 37:326-340.

Carvell, C., W. R. Meek, R. F. Pywell, D. Goulson, and M. Nowakowski. 2007. Comparing the efficacy of agrienvironment schemes to enhance bumble bee abundance and diversity on arable field margins. Journal of Applied Ecology 44:29-40.
Carvell, C., W. C. Jordan, A. F. G. Bourke, R. Pickles, J. W. Redhead, and M. S. Heard. 2012. Molecular and spatial analyses reveal links between colony-specific foraging distance and landscape-level resource availability in two bumble bee species. Oikos 121:734-742.

Chapman, R. E., J. Wang, and A. F. G. Bourke. 2003. Genetic analysis of spatial foraging patterns and resource sharing in bumble bee pollinators. Molecular Ecology 12:2801-2808

Charman, T. G., J. Sears, R. E. Green, and A. F. G. Bourke. 2010. Conservation genetics, foraging distance and nest density of the scarce Great Yellow Bumble bee (Bombus distinguendus). Molecular Ecology 19:2661-2674.

Cranmer, L., D. McCollin, and J. Ollerton. 2012. Landscape structure influences pollinator movements and directly affects plant reproductive success. Oikos 121:562-568.

Cresswell, J. E., J. L. Osborne, and D. Goulson. 2000. An economic model of the limits to foraging range in central place foragers with numerical solutions for bumble bees. Ecological Entomology 25:249-255.

Darvill, B., M. E. Knight, and D. Goulson. 2004. Use of genetic markers to quantify bumble bee foraging range and nest density. Oikos 107:471-478.

Dramstad, W. E. 1996. Do bumble bees (Hymenoptera: Apidae) really forage close to their nests? Journal of Insect Behavior 9:163-182.

Dramstad, W., and G. Fry. 1995. Foraging activity of bumble bees (Bombus) in relation to flower resources on arable land. Agriculture, Ecosystems \& Environment 53:123-135.

Dreier, S., J. W. Redhead, I. Warren, A. F. G. Bourke, M. S. Heard, W. C. Jordan, S. Sumner, J. Wang, and C. Carvell. 2014a. Microsatellite genotype data for five species of bumble bee across an agricultural landscape in Buckinghamshire, UK. NERC Environmental Information Data Centre.

Dreier, S., J. W. Redhead, I. A. Warren, A. F. G. Bourke, M. S. Heard, W. C. Jordan, S. Sumner, J. Wang, and C. Carvell. 2014b. Fine-scale spatial genetic structure of common and declining bumble bees across an agricultural landscape. Molecular Ecology 23:3384-3395.

Dukas, R., and L. Edelstein-Keshet. 1998. The spatial distribution of colonial food provisioners. Journal of Theoretical Biology 190:121-134.

Ellis, J. S., M. E. Knight, C. Carvell, and D. Goulson. 2006. Cryptic species identification: a simple diagnostic tool for discriminating between two problematic bumble bee species. Molecular Ecology Notes 6:540-542.

Garibaldi, L. A., et al. 2014. From research to action: enhancing crop yield through wild pollinators. Frontiers in Ecology and the Environment 12:439-447.

Garratt, M. P. D., D. J. Coston, C. L. Truslove, M. G. Lappage, C. Polce, R. Dean, J. C. Biesmeijer, and S. G. Potts. 2014. The identity of crop pollinators helps target conservation for improved ecosystem services. Biological Conservation 169:128-135.

Goulson, D. 2010. Bumble bees. Pages 415-429 in N. Maclean, editor. Silent Summer: The State of Wildlife in Britain and Ireland. Cambridge University Press, Cambridge.

Goulson, D., M. E. Hanley, B. Darvill, J. S. Ellis, and M. E. Knight. 2005. Causes of rarity in bumble bees. Biological Conservation 122:1-8.

Goulson, D., G. C. Lye, and B. Darvill. 2008. Decline and conservation of bumble bees. Annual Review of Entomology 53:191-208.

Goulson, D., O. Lepais, S. O'Connor, J. L. Osborne, R. A. Sanderson, J. Cussans, L. Goffe, and B. Darvill. 2010. Effects of land use at a landscape scale on bumble bee 
nest density and survival. Journal of Applied Ecology 47:1207-1215.

Greenleaf, S., N. Williams, R. Winfree, and C. Kremen. 2007. Bee foraging ranges and their relationship to body size. Oecologia 153:589-596.

Hagen, M., M. Wikelski, and W. D. Kissling. 2011. Space use of bumble bees (Bombus spp.) revealed by radio-tracking. PLoS One 6:e19997.

Herrmann, F., C. Westphal, R. F. A. Moritz, and I. SteffanDewenter. 2007. Genetic diversity and mass resources promote colony size and forager densities of a social bee (Bombus pascuorum) in agricultural landscapes. Molecular Ecology 16:1167-1178.

Holehouse, K. A., R. L. Hammond, and A. F. G. Bourke. 2003. Non-lethal sampling of DNA from bumble bees for conservation genetics. Insectes Sociaux 50:277-285.

Holzschuh, A., C. F. Dormann, T. Tscharntke, and I. SteffanDewenter. 2011. Expansion of mass-flowering crops leads to transient pollinator dilution and reduced wild plant pollination. Proceedings of the Royal Society B: Biological Sciences 278:3444-3451.

Jha, S., and C. Kremen. 2013. Resource diversity and landscapelevel homogeneity drive native bee foraging. Proceedings of the National Academy of Sciences 110:555-558.

Knight, M. E., A. P. Martin, S. Bishop, J. L. Osborne, R. J. Hale, R. A. Sanderson, and D. Goulson. 2005. An interspecific comparison of foraging range and nest density of four bumble bee (Bombus) species. Molecular Ecology 14:1811-1820.

Knight, M. E., J. L. Osborne, R. A. Sanderson, R. J. Hale, A. P. Martin, and D. Goulson. 2009. Bumble bee nest density and the scale of available forage in arable landscapes. Insect Conservation and Diversity 2:116-124.

Kovács-Hostyánszki, A., S. Haenke, P. Batáry, B. Jauker, A. Báldi, T. Tscharntke, and A. Holzschuh. 2013. Contrasting effects of mass-flowering crops on bee pollination of hedge plants at different spatial and temporal scales. Ecological Applications 23:1938-1946.

Krauss, J., et al. 2010. Habitat fragmentation causes immediate and time-delayed biodiversity loss at different trophic levels. Ecology Letters 13:597-605.

Lonsdorf, E., C. Kremen, T. Ricketts, R. Winfree, N. Williams, and S. Greenleaf. 2009. Modelling pollination services across agricultural landscapes. Annals of Botany 103:1589-1600.

Moser, D., H. Zechmeister, C. Plutzar, N. Sauberer, T. Wrbka, and G. Grabherr. 2002. Landscape patch shape complexity as an effective measure for plant species richness in rural landscapes. Landscape Ecology 17:657-669.

Osborne, J. L., S. J. Clark, R. J. Morris, I. H. Williams, J. R. Riley, A. D. Smith, D. R. Reynolds, and A. S. Edwards. 1999. A landscape-scale study of bumble bee foraging range and constancy, using harmonic radar. Journal of Applied Ecology 36:519-533.

Osborne, J. L., A. P. Martin, N. L. Carreck, J. L. Swain, M. E. Knight, D. Goulson, R. J. Hale, and R. A. Sanderson. 2008. Bumble bee flight distances in relation to the forage landscape. Journal of Animal Ecology 77:406-415.

Persson, A. S., and H. G. Smith. 2013. Seasonal persistence of bumble bee populations is affected by landscape context. Agriculture, Ecosystems \& Environment 165:201-209.

Prys-Jones, O. E., and S. A. Corbet. 1991. Bumble bees. The Richmond Publishing Co., Ltd, Slough.

R Core Team. 2013. R: A language and environment for statistical computing. R Foundation for Statistical Computing, Vienna, Austria.
Raine, N. E., D. K. Rossmo, and S. C. Le Comber. 2009. Geographic profiling applied to testing models of bumblebee foraging. Journal of The Royal Society Interface 6:307-319.

Redhead, J. W., R. F. Pywell, P. E. Bellamy, R. K. Broughton, R. A. Hill, and S. A. Hinsley. 2013. Great tits Parus major and blue tits Cyanistes caeruleus as indicators of agrienvironmental habitat quality. Agriculture, Ecosystems \& Environment 178:31-38.

Riitters, K. H., R. V. O’Neill, C. T. Hunsaker, J. D. Wickham, D. H. Yankee, S. P. Timmins, K. B. Jones, and B. L. Jackson. 1995. A factor analysis of landscape pattern and structure metrics. Landscape Ecology 10:23-39.

Rundlof, M., H. Nilsson, and H. G. Smith. 2008. Interacting effects of farming practice and landscape context on bumble bees. Biological Conservation 141:417-426.

Schmid-Hempel, R., and P. Schmid-Hempel. 1998. Colony performance and immunocompetence of a social insect, Bombus terrestris, in poor and variable environments. Functional Ecology 12:22-30.

Stanley, D. A., M. E. Knight, and J. C. Stout. 2013. Ecological variation in response to mass-flowering oilseed rape and surrounding landscape composition by members of a cryptic bumble bee complex. PLoS One 8:e65516.

Stewart, L. C., R. J. Hale, and M. L. Hale. 2010. Speciesspecific primers for the molecular identification of cryptic Bombus species in New Zealand. Conservation Genetics 11:1207-1209.

Stout, J. C., J. A. Allen, and D. Goulson. 2000. Nectar robbing, forager efficiency and seed set: bumble bees foraging on the self incompatible plant Linaria vulgaris (Scrophulariaceae). Acta Oecologica 21:277-283.

Suzuki, Y., L. G. Kawaguchi, and Y. Toquenaga. 2007. Estimating nest locations of bumble bee Bombus ardens from flower quality and distribution. Ecological Research 22:220-227.

Truett, G. E., P. Heeger, R. L. Mynatt, A. A. Truett, J. A. Walker, and M. L. Warman. 2000. Preparation of PCRquality mouse genomic DNA with hot sodium hydroxide and tris (HotSHOT). BioTechniques 29(52, 54.

Walther-Hellwig, K., and R. Frank1. 2000. Foraging habitats and foraging distances of bumble bees, Bombus spp. (Hym., apidae), in an agricultural landscape. Journal of Applied EntomologyZeitschrift Fur Angewandte Entomologie 124:299-306.

Wang, J. 2004. Sibship reconstruction from genetic data with typing errors. Genetics 166:1963-1979.

Westphal, C., I. Steffan-Dewenter, and T. Tscharntke. 2003. Mass flowering crops enhance pollinator densities at a landscape scale. Ecology Letters 6:961-965.

Westphal, C., I. Steffan-Dewenter, and T. Tscharntke. 2006a. Bumble bees experience landscapes at different spatial Scales: possible implications for coexistence. Oecologia 149:289-300.

Westphal, C., I. Steffan-Dewenter, and T. Tscharntke. 2006b. Foraging trip duration of bumble bees in relation to landscape-wide resource availability. Ecological Entomology 31:389-394.

Westphal, C., I. Steffan-Dewenter, and T. Tscharntke. 2009. Mass flowering oilseed rape improves early colony growth but not sexual reproduction of bumble bees. Journal of Applied Ecology 46:187-193.

Williams, P., and J. L. Osborne. 2009. Bumble bee vulnerability and conservation world-wide. Apidologie 40:367-387.

Wood, T. J., J. M. Holland, W. O. Hughes, and D. Goulson. 2015. Targeted agri-environment schemes significantly improve the population size of common farmland bumble bee species. Molecular Ecology 24:1668-1680. 


\section{SUPPORTING INFORMATION}

Additional supporting information may be found in the online version of this article at http://onlinelibrary.wiley.com/ doi/10.1890/15-0546/suppinfo

\section{Data Availability}

Microsatellite genotype data for five species of bumble bee across an agricultural landscape in Buckinghamshire, UK. NERC Environmental Information Data Centre(EIDC): DOI: 10.5285/6a408415-0575-49c6-af69-b568e343266d.

Location data of worker bumble bees across an agricultural landscape in Buckinghamshire, UK. NERC Environmental Information Data Centre (EIDC): DOI: 10.5285/a60f52b8-0f9f-44f6-aca4-861cb461a0eb.

Map of land-use/land-cover and floral cover across an arable landscape in Buckinghamshire, UK. NERC Environmental Information Data Centre(EIDC): DOI: 10.5285/0667cf06-f2c3-45c1-a80a-e48539b52427. 